\title{
Location of Saturn's northern infrared aurora determined from Cassini VIMS images
}

\author{
S. V. Badman, ${ }^{1}$ N. Achilleos, ${ }^{2}$ K. H. Baines,${ }^{3}$ R. H. Brown, ${ }^{4}$ E. J. Bunce,${ }^{5}$ \\ M. K. Dougherty, ${ }^{6}$ H. Melin, ${ }^{5}$ J. D. Nichols, ${ }^{5}$ and T. Stallard ${ }^{5}$ \\ Received 14 November 2010; revised 22 December 2010; accepted 3 January 2011; published 4 February 2011.
}

[1] The location of Saturn's northern infrared aurora is described in detail using 12 selected images acquired by Cassini VIMS during 2006-2008. Bright main oval arcs and prevalent polar features are displayed, which do not exhibit a preferred local time or spatial extent. The average equatorward limit of the aurora was determined using a best fit circle method. The average circle has a radius of $16.4 \pm$ $0.2^{\circ}$ latitude, centred $1.6^{\circ}$ anti-sunward of Saturn's pole. The low standard deviation of the fitted circle radii $\left(<1^{\circ}\right)$ indicates the relative stability of the equatorward auroral boundary. For the first time we show that the average location of the infrared oval is similar to that of the ultraviolet oval, suggesting that the auroral emissions at different wavelengths are very likely produced by a common driver despite the distinct emission mechanisms. Citation: Badman, S. V., N. Achilleos, K. H. Baines, R. H. Brown, E. J. Bunce, M. K. Dougherty, H. Melin, J. D. Nichols, and T. Stallard (2011), Location of Saturn's northern infrared aurora determined from Cassini VIMS images, Geophys. Res. Lett., 38, L03102, doi:10.1029/ 2010GL046193.

\section{Introduction}

[2] Observations of Saturn's aurora provide a unique means of remote sensing the planet's dynamic magnetosphere. Studies of the southern ultraviolet (UV) emissions, e.g., by the Hubble Space Telescope (HST) have revealed that the aurora generally takes the form of an oval of radius $\sim 15^{\circ}$ co-latitude centred roughly on the pole. Nevertheless, the morphology, brightness and location of the aurora are variable and have exhibited dependences on the impinging solar wind and the pulsing of Saturn's kilometric radiation [Clarke et al., 2005; Crary et al., 2005; Nichols et al., 2010]. Interpretation of auroral images combined with modelling and in situ measurements have demonstrated that the main oval emissions are generated by a field-aligned current system associated with the boundary between open and closed magnetic field lines [Cowley et al., 2004; Badman et al., 2006; Bunce et al., 2008a]. Ground-based infrared (IR) observations

\footnotetext{
${ }^{1}$ Institute of Space and Astronautical Science, Japan Aerospace Exploration Agency, Sagamihara, Japan.

${ }^{2}$ Atmospheric Physics Laboratory, Centre for Planetary Sciences, University College London, London, UK.

${ }^{3}$ SSEC, University of Wisconsin-Madison, Madison, Wisconsin, USA.

${ }^{4}$ Lunar and Planetary Laboratory, University of Arizona, Tucson, Arizona, USA.

${ }^{5}$ Department of Physics and Astronomy, University of Leicester, Leicester, UK.

${ }^{6}$ Blackett Laboratory, Imperial College London, London, UK.
}

Copyright 2011 by the American Geophysical Union. 0094-8276/11/2010GL046193 have also indicated the presence of an auroral oval around Saturn's poles, but accurate measurements of the latitudinal position of the emission could not be made from those data because of the low spatial resolution and inaccuracies in locating the position on the planet. Infrared emissions are sometimes detected within the main oval, and can extend across the polar region and reach intensities comparable to the main oval emissions [Stallard et al., 2007]. The bright IR polar region has also been detected in images taken by the Visual and Infrared Mapping Spectrometer (VIMS) [Brown et al., 2004] onboard Cassini, as will be discussed below.

[3] Upon impacting the upper atmosphere, incident electrons undergo several interactions to generate auroral emissions across a range of wavelengths. The strongest UV emissions are $H$ Lyman- $\alpha$ and $H_{2}$ Lyman- and Werner-band transitions, following excitation by electron impact [e.g., Broadfoot et al., 1981]. To generate IR emissions, however, the electrons ionize atmospheric $\mathrm{H}_{2}$ to form $\mathrm{H}_{2}^{+}$which then reacts with $\mathrm{H}_{2}$ to produce the $H_{3}^{+}$ionized molecule. Rovibrational transitions of the $H_{3}^{+}$molecule produce the observed IR auroral emissions [Drossart et al., 1989]. The majority of the IR and UV aurora are thought to be emitted from similar altitudes above the homopause [e.g., Stallard et al., 2007; Gérard et al., 2009], suggesting their basic morphologies could match. However, factors such as the indirect production of $\mathrm{H}_{3}^{+}$and the temperature dependence of its emissions complicate this initial assumption. For example, Stallard et al. [2008] identified bright polar emissions in VIMS images of the IR aurora and suggested they could be attributed to either a region of raised atmospheric temperature (which would lead to intensified IR but not UV emissions), or to the nature of the magnetospheric field lines and particles mapping to the aurora. It is clear therefore that while the UV and IR aurora are similar in many ways, they also exhibit intriguing differences. The purpose of this study is to characterise one of the fundamental properties of the IR aurora, its location, to help constrain the relationship. As Saturn's northern aurora has only recently been visible from the Earth as Saturn passed through equinox, they have not yet been studied in detail. This study therefore focusses on analysis of the northern main oval aurora under winter conditions.

\section{Description of Auroral Images}

[4] This study employs twelve images of Saturn's northern IR aurora acquired by Cassini VIMS during 2006-2008. The start times of each image are listed in Table 1. These images were selected because the VIMS field of view (FOV) covered a large portion of the northern auroral region, and the accumulation time was long (tens of minutes) such that auroral emissions were distinguished and could be fitted by the 
Table 1. Details of VIMS Images Included in This Study

\begin{tabular}{|c|c|c|c|c|c|}
\hline \multirow[b]{2}{*}{ Figure } & \multirow[b]{2}{*}{ Start Time (UT) } & \multirow[b]{2}{*}{ Accum. Time (min) } & \multicolumn{3}{|c|}{ Best Fit Circle } \\
\hline & & & Centre x (deg) & Centre y (deg) & Radius (deg) \\
\hline $1 \mathrm{a}$ & $200631414: 18$ & 72 & -0.4 & 0.7 & $17.9 \pm 1.0$ \\
\hline $1 b$ & 2007144 04:43 & 67 & -3.3 & 1.4 & $16.8 \pm 1.4$ \\
\hline $1 \mathrm{c}$ & $200716012: 40$ & 46 & -2.1 & 0.2 & $17.3 \pm 1.2$ \\
\hline $1 d$ & $2008037 \quad 12: 12$ & 65 & -0.5 & -0.5 & $15.8 \pm 1.4$ \\
\hline $1 \mathrm{e}$ & 2008109 08:26 & 65 & -0.1 & -1.0 & $15.7 \pm 1.2$ \\
\hline $1 \mathrm{f}$ & $200812906: 41$ & 68 & -0.7 & -0.5 & $15.4 \pm 1.3$ \\
\hline $1 \mathrm{~g}$ & $200820014: 49$ & 65 & -2.1 & -0.8 & $16.2 \pm 1.3$ \\
\hline $1 \mathrm{~h}$ & $200820113: 38$ & 65 & -2.9 & -1.8 & $15.9 \pm 1.6$ \\
\hline $1 \mathrm{i}$ & 2008207 13:19 & 65 & -2.2 & -0.9 & $15.8 \pm 1.2$ \\
\hline $1 \mathrm{j}$ & 2008208 02:39 & 68 & -1.0 & -1.0 & $15.8 \pm 1.0$ \\
\hline $1 \mathrm{k}$ & 2008334 21:02 & 67 & -1.3 & -0.1 & $17.2 \pm 1.0$ \\
\hline 11 & 2008335 01:59 & 67 & -2.3 & 0.8 & $17.1 \pm 1.3$ \\
\hline Average & & & -1.6 & -0.3 & $16.4 \pm 0.2$ \\
\hline Std. dev. & & & 1.1 & 0.9 & 0.8 \\
\hline
\end{tabular}

boundary algorithm described below. The VIMS IR channel covers the wavelength range $0.85-5.1 \mu \mathrm{m}$ in 256 spectral bins. A full spectrum is taken at each pixel position in the FOV in turn (where 1 pixel $=0.5 \times 0.5 \mathrm{mrad}$ and the maximum FOV is $64 \times 64$ pixels), such that a full 2D spatial image takes typically tens of minutes to construct. Each image shown here is a sum of two spectral bins containing $H_{3}^{+}$auroral wavelengths, with two non-auroral 'background' spectral bins subtracted. Each bin has an approximately Gaussian throughput, with the two auroral bins centred on $3.532 \mu \mathrm{m}$ and $3.667 \mu \mathrm{m}$ and the two background bins centred on $3.565 \mu \mathrm{m}$ and $3.702 \mu \mathrm{m}$, with FWHM of $0.0161 \mu \mathrm{m}$, $0.0184 \mu \mathrm{m}, 0.0165 \mu \mathrm{m}$ and $0.0188 \mu \mathrm{m}$, respectively.

[5] The images are shown in Figure 1, where they have been projected onto a planetocentric polar grid using an estimated peak emission height of $1000 \mathrm{~km}$ above the $1 \mathrm{bar}$ reference spheroid [Gérard et al., 2009]. The orientation is such that the observer is looking down onto the pole with the sunward direction (12 LT) at the bottom, dawn (06 LT) to the left and dusk (18 LT) to the right. The LT orientation is determined using Cassini trajectory information. The overlaid yellow grid marks latitudes at intervals of $10^{\circ}$ and the noon-midnight and dawn-dusk meridians. The data is colourcoded on a red-white scale according to emission intensity while the grey-shaded region shows the areas outside the VIMS FOV. The absolute intensities of the emissions are not presented here because calibration uncertainties remain in their determination. The effects of the changing FOV are evident in the variable size of the pixels when projected onto the polar grid, however these regions are avoided in our analysis as will be described in Section 3 .

[6] In the first image shown in Figure 1, taken on day 314 of 2006, the aurora formed a narrow arc on the dawnside at $\sim 17^{\circ}$ co-latitude. The next two images from days 144 and 160 of 2007 are examples of the very bright polar emission previously discussed by Stallard et al. [2008]. In the image on day 144 (Figure $1 \mathrm{~b}$ ) the emission appeared to extend across the pole from the dawnside to approximately $10^{\circ}$ co-latitude on the duskside, with a dark gap between it and the somewhat fainter main oval at lower latitudes. There was a discontinuity in the main oval at around $16 \mathrm{LT}$, changing from $\sim 13^{\circ}$ to $\sim 17^{\circ}$ co-latitude from noon to dusk. On day 160 (Figure 1c) the polar region was infilled by very bright emission from the limit of the FOV on the dawnside out to $\sim 10^{\circ}$ co-latitude on the duskside. Again, there was a fainter region a few degrees wide inside the main oval. The emission extended to $\sim 16^{\circ}$ co-latitude at noon, increasing to $\sim 19^{\circ}-20^{\circ}$ co-latitude at dusk and post-dusk. Figures 1d-1f taken on days 37, 109 and 129 of 2008 show very different morphologies to those just described. In each the aurora formed a narrow arc with faint polar emission. These auroral arcs were located at co-latitudes $15^{\circ}-16^{\circ}$ in each image and were brightest on the dayside on day 37 , shifting $\sim 2^{\circ}$ poleward around noon, formed equally bright arcs on the pre-noon and nightside regions on day 109 , and were only present in the dawn sector on day 129.

[7] The next images in Figures 1g and 1h, taken on days 200 and 201 of 2008 , both exhibited a narrow $\left(\sim 2^{\circ}\right)$, bright arc of emission at $\sim 15^{\circ}$ co-latitude on the dawnside, which became fainter around noon. A bright feature was observed at higher latitude $\left(\sim 7^{\circ}-12^{\circ}\right.$ co-latitude) around dusk. On day 201 this feature became fainter post-dusk and approached the main oval emissions at $\sim 20 \mathrm{LT}$. The image taken on day 2072008 (Figure 1i) showed a narrow auroral arc at $\sim 16^{\circ}$ co-latitude pre-dawn. The emission was patchy in the dawn to noon sector then brightened post-noon at similar latitudes. Diffuse emission was also detected in the polar region, with two particularly bright spots at $\sim 8 \mathrm{LT}, 7^{\circ}$ co-latitude and 11-13 LT, $\sim 8^{\circ}$ co-latitude. Thirteen hours later (Figure 1j) the narrow dawnside arc of emission was clearly defined from the edge of the FOV post-midnight to pre-noon. A post-noon brightening was also observed, but at higher latitude and duskward of that in the previous image. A bright, diffuse spot was observed in the dawn-to-noon sector poleward of $10^{\circ}$ co-latitude.

[8] The final two images included in this study, shown in Figures $1 \mathrm{k}$ and 11 , were taken at 21 UT on day 334 of 2008, and $5 \mathrm{~h}$ later at $02 \mathrm{UT}$ on day 335. The image in Figure $1 \mathrm{k}$ displays an auroral arc at $\sim 15^{\circ}-18^{\circ}$ co-latitude across the nightside, with faint emission around the dawn-dusk meridian. Two bright patches were identified on the edges of the FOV at $\sim 9$ LT and 16 LT. In the later image (Figure 11) the arcs were also observed at $\sim 15^{\circ}-18^{\circ}$ co-latitude, but were brightest at dawn and dusk and faint at midnight. There were no polar emissions of comparable intensity to the main oval. Overall, the IR auroral morphologies observed in these images demonstrate variability in both form and location. Bright arcs were commonly observed at all local times as well as diffuse 


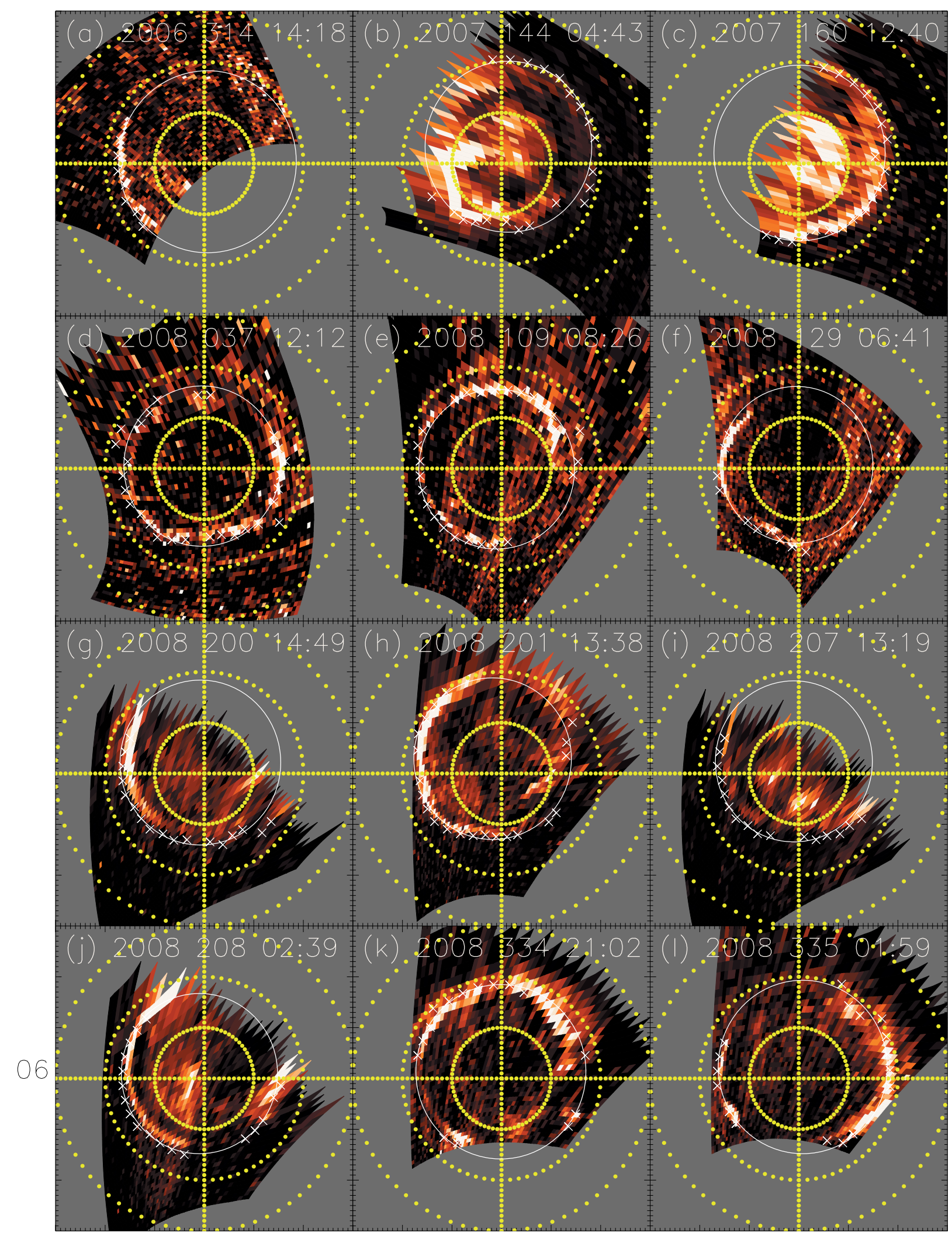

12

Figure 1. (a-i) Images of Saturn's northern IR aurora acquired by Cassini VIMS. The date (year and day of year) and start time of each image are printed at the top of each image. The images have been polar projected to an altitude of $1000 \mathrm{~km}$ above the 1 bar reference spheroid and are orientated with the sunward direction at the bottom, dawn to the left and dusk to the right, labelled on Figure 1j. The grey-shaded regions are areas outside the VIMS FOV. The yellow grid marks the noonmidnight and dawn-dusk meridians, and latitudes at intervals of 10 degrees. The white crosses and circles mark the boundary points and best fit to the equatorward auroral boundary for each image. 


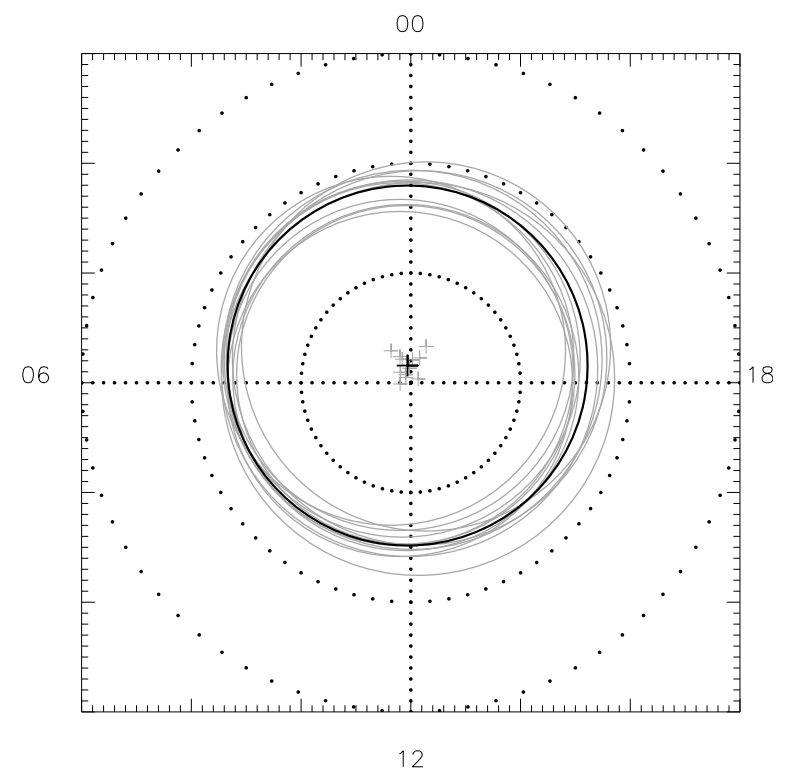

Figure 2. Polar view of the best-fit auroral boundary circles for each image (grey) and the average circle (black). The individual and average circle centres are marked by the grey and black crosses, respectively.

polar emissions. The polar emissions varied from localised spots to large-scale infilling. The main oval emissions extended to co-latitudes $\sim 14^{\circ}-20^{\circ}$ and will be further characterised in the next section.

\section{Boundary Determination and Fitting}

[9] The equatorward boundary of the auroral emissions was examined rather than the poleward boundary for the following reasons. First, the IR aurora is emitted from an ionospheric layer of finite height. VIMS usually observes the aurora from sub-auroral latitudes and the height of the emission layer therefore modifies the apparent poleward edge of the aurora. In addition, the definition of the poleward boundary is complicated by the occasional presence of bright emission at higher latitudes than the main oval as described above, the origin of which is currently under investigation. Finally, characterisation of the equatorward boundary allows direct comparison of the location of the IR auroral boundary with that identified for the northern UV aurora by Nichols et al. [2009].

[10] To determine the typical extent of the IR aurora, the equatorward boundary of the emission was marked at intervals of $10^{\circ}$ longitude, where a sharp boundary could be determined within the FOV. This was performed using an automated procedure which placed the boundary at the co-latitude where the minimum (negative) gradient in intensity occurred along each meridian. These points are marked by the white crosses in Figure 1. Given the variable pixel size, the boundaries were determined with $1^{\circ}$ accuracy. Emissions at the edge of the FOV where the pixels were very stretched by the viewing geometry (e.g., the midnight region of Figure $1 \mathrm{~h}$ ), or those where instrumental artefacts were not well removed (e.g., the dusk region of Figure 1f) were not included. For each image the boundary could be determined at a limited set of longitudes, depending on the FOV and the auroral morphology. A maximum of ten values out of a possible twelve were measured in the dawn region (at longitudes $70^{\circ}$ and $130^{\circ}$ using a sun-relative definition of longitude increasing eastward from midnight). Only three or four points could be marked around midnight $\left(310^{\circ}\right.$ to $\left.20^{\circ}\right)$. Similar numbers of boundary points could be marked on both the dawn and dusk sides (totals of 125 on the dawnside versus 100 on the duskside). Past analysis of UV images has revealed that emissions are significantly more often detected on the dawnside than the dusk [e.g., Badman et al., 2006]. It is interesting therefore to note that a more symmetric distribution is identified here in the IR. However, such investigation is inhibited by the limited FOV, i.e., both dawn and dusk sides are not always visible in the same image (see Figure 1). Although this study is based on a subset of images, the standard deviation (a measure of the variability) of the 225 individual boundary points that went into the analysis is $2.1^{\circ}$, which is comparable to the value derived for a larger set of UV auroral images [Badman et al., 2006], suggesting that the results shown here are representative of the variability of a larger data set.

[11] A best fit circle was derived for the boundary points detected for each image. These circles are plotted in white in Figures 1a-1i. As expected for a complex dynamic phenomenon, the circles are not a perfect fit to the auroral emissions, but do provide a good smoothed boundary approximation as can be judged from Figure 1. The radius of each circle, the coordinates of its centre, and the associated standard errors (derived from the $\pm 0.5^{\circ}$ uncertainty in the marked boundary positions and the calculated error in fitting the circle to the points) are listed in Table 1 . The best-fit circles are plotted together on a polar grid by the grey circles in Figure 2 . The centres of the circles are also plotted by the grey crosses. This view shows that the location of the equatorward boundary showed least variation in the pre-dawn sector, varying between $15^{\circ}$ and $18^{\circ}$ co-latitude, and the highest variation in the pre-dusk sector: $13^{\circ}-18^{\circ}$ co-latitude.

[12] The centre points of the best fit circles were all displaced toward the nightside ( $\mathrm{x}$ direction) by between $\sim 0.3^{\circ}$ and $3.3^{\circ}$ (see Table 1). The dawn-dusk offset (y positive to dusk) of the circle centre varied by up to $1.8^{\circ}$ dawnward and $1.4^{\circ}$ duskward. Averaging over the derived best fit circles, the mean circle was found to have a radius of $16.4 \pm 0.2^{\circ}$ with a centre position of $1.6^{\circ}$ anti-sunward and $0.3^{\circ}$ towards dawn. This average circle and its centre are marked in black on Figure 2.

\section{Discussion}

[13] The auroral images presented in this study reveal a variety of morphologies, however the equatorward extent of the emissions can be well approximated by a circle. The average radius of the best fit circles is $16.4 \pm 0.2^{\circ}$ and the standard deviation of this value is $0.8^{\circ}$. The low standard deviation demonstrates the relative stability of the equatorward auroral boundary. Following the discussion in the introduction, while the poleward boundary of the aurora has been shown to lie at the boundary between open and closed field lines and thus changes position over a large range of latitudes depending on the solar wind interaction, the equatorward boundary of the aurora maps deeper inside the magnetosphere, close to the outer edge of the ring current region according to the most up-to-date models, and is more 
stable over time [Badman et al., 2005, 2006; Bunce et al., 2008b].

[14] Nichols et al. [2009] used a similar method of circlefitting to determine the average equatorward boundary of Saturn's northern UV aurora from HST images taken in early 2009. They found a circle radius of $16.3 \pm 0.6^{\circ}$, which is the same, within the ranges of standard errors quoted, as the value derived here for the IR aurora. The northern IR auroral oval is typically offset towards midnight by $\sim 1.6^{\circ}$, such that the average equatorward boundary of the aurora is found to lie at $\sim 15^{\circ}$ co-latitude on the dayside, as suggested for the UV aurora by Badman et al. [2006]. The magnitude of the anti-sunward offset identified in this study is comparable to that determined for the southern UV aurora by Nichols et al. [2008]. These correspondences of the typical locations of the IR and UV aurora suggest that the emissions are driven by a common process; e.g., they occur at the ionospheric footprint of the same field lines, despite the different emission processes.

[15] In the UV the radius of the southern auroral oval has been found to be $\sim 1.5^{\circ}$ larger than in the north [Nichols et al., 2009] owing to the interhemispheric asymmetry in the Saturn's internal magnetic field [Dougherty et al., 2005] and this effect should therefore be expected in the IR aurora as well. Nichols et al. [2008] also identified an oscillation in the southern UV auroral oval by examining the motion of the centre of the best fit circle. Such comparative investigations using the IR auroral data set remain topics for future work, but we note here that the standard deviations of the ' $x$ ' (sunward) and ' $y$ ' (duskward) circle centre positions are $1.1^{\circ}$ and $0.9^{\circ}$, respectively, suggesting that significant variations are present that may be ordered by a planetary period oscillation, although the overall ranges in the ' $x$ ' and ' $y$ ' positions identified (see Table 1 and Figure 2) are smaller than those determined for the southern UV aurora. As the results presented here apply for northern winter and solar minimum conditions it will be very useful to compare with analysis of images taken during different seasons in both hemispheres.

[16] In addition to the main oval it is also of interest to consider the occurrence of the polar emissions. Small-scale 'spot' features (e.g., in the dawn-noon sector of Figure 1i) were observed, as well as broad diffuse patches (e.g., the dawn sector of Figure 1h), and intense large-scale infilling (Figures 1b and 1c). The polar emissions discussed in this study did not exhibit a preferred LT, latitude or size. Like the main oval, they were transient on timescales of $5 \mathrm{~h}$ or less (Figures $1 \mathrm{k}$ and 11), and even as short as $45 \mathrm{~min}$ [Stallard et al., 2008]. Overall it is clear that the region poleward of the main oval was more intense in the IR than the lower latitude region. Further analysis is underway to develop understanding of the generation of the polar emissions in relation to the main oval.

\section{Conclusions}

[17] We have presented images of Saturn's northern IR aurora acquired by Cassini VIMS during northern winter. A variety of morphologies are observed including intense arcs at all local times, diffuse patches, and both small- and largescale bright polar emissions. The average equatorward boundary of the main oval emissions was determined using a circle-fitting method. The average best fit circle has a radius of $16.4 \pm 0.2^{\circ}$ latitude and is offset by $1.6^{\circ}$ towards midnight.
This is the first analysis of the location of Saturn's IR aurora, made possible by the Cassini VIMS data set. The average size of the IR auroral oval is close to that derived for the northern UV emissions from HST images, and its location at $\sim 15^{\circ}$ co-latitude on the dayside agrees with predictions made for the northern aurora based on analysis of the UV southern oval [Badman et al., 2006; Nichols et al., 2009]. These results imply a common driver (e.g., field-aligned current system) for the main oval emissions at different wavelengths. The anti-sunward offset of the circle from the pole is similar to that observed for the southern UV oval, and is possibly due to solar wind pressure on the dayside [Clarke et al., 2005; Nichols et al., 2008]. These results thus provide an important foundation for understanding the IR morphologies and emission mechanisms, as well as the relationship with the UV observations. These results and other details will be expanded on by future analysis of simultaneous multi-wavelength auroral observations by Cassini under different seasonal conditions.

[18] Acknowledgments. This work was facilitated by the Cassini MAG-VIMS collaboration. SVB thanks D. Lytle (U. Arizona) and D.J. Andrews (U. Leicester) for assistance with VIMS and SPICE. Support was provided by the following grants: ST/H002480/1 (JDN, TS), ST/ G0022223/1 (TS, HM), an RCUK fellowship (TS). The authors acknowledge the support of ISSI, as this study was discussed by ISSI International Team 178

\section{References}

Badman, S. V., E. J. Bunce, J. T. Clarke, S. W. H. Cowley, J.-C. Gérard, D. Grodent, and S. E. Milan (2005), Open flux estimates in Saturn's magnetosphere during the January 2004 Cassini-HST campaign, and implications for reconnection rates, J. Geophys. Res., 110, A11216, doi:10.1029/2005JA011240.

Badman, S. V., et al. (2006), A statistical analysis of the location and width of Saturn's southern auroras, Ann. Geophys., 24(12), 3533-3545.

Broadfoot, A. L., et al. (1981), Extreme ultraviolet observations from Voyager-1 encounter with Saturn, Science, 212(4491), 206-211.

Brown, R. H., et al. (2004), The Cassini Visual and Infrared Mapping Spectrometer (VIMS) investigation, Space Sci. Rev., 115(1-4), 111-168.

Bunce, E. J., et al. (2008a), Origin of Saturn's aurora: Simultaneous observations by Cassini and the Hubble Space Telescope, J. Geophys. Res., 113, A09209, doi:10.1029/2008JA013257.

Bunce, E. J., C. S. Arridge, S. W. H. Cowley, and M. K. Dougherty (2008b), Magnetic field structure of Saturn's dayside magnetosphere and its mapping to the ionosphere: Results from ring current modeling, J. Geophys. Res., 113, A02207, doi:10.1029/2007JA012538.

Clarke, J. T., et al. (2005), Morphological differences between Saturn's ultraviolet aurorae and those of Earth and Jupiter, Nature, 433(7027), 717-719.

Cowley, S. W. H., E. J. Bunce, and J. M. O'Rourke (2004), A simple quantitative model of plasma flows and currents in Saturn's polar ionosphere, J. Geophys. Res., 109, A05212, doi:10.1029/2003JA010375.

Crary, F., et al. (2005), Solar wind dynamic pressure and electric field as the main factors controlling Saturn's aurorae, Nature, 433(7027), $720-722$.

Dougherty, M. K., et al. (2005), Cassini magnetometer observations during Saturn orbit insertion, Science, 307(5713), 1266-1270.

Drossart, P., et al. (1989), Detection of $H_{3}^{+}$on Jupiter, Nature, 340(6234), 539-541.

Gérard, J.-C., B. Bonfond, J. Gustin, D. Grodent, J. T. Clarke, D. Bisikalo, and V. Shematovich (2009), Altitude of Saturn's aurora and its implications for the characteristic energy of precipitated electrons, Geophys. Res. Lett., 36, L02202, doi:10.1029/2008GL036554.

Nichols, J. D., J. T. Clarke, S. W. H. Cowley, J. Duval, A. J. Farmer, J.-C. Gérard, D. Grodent, and S. Wannawichian (2008), Oscillation of Saturn's southern auroral oval, J. Geophys. Res., 113, A11205, doi:10.1029/ 2008JA013444

Nichols, J. D., et al. (2009), Saturn's equinoctial auroras, Geophys. Res. Lett., 36, L24102, doi:10.1029/2009GL041491.

Nichols, J. D., B. Cecconi, J. T. Clarke, S. W. H. Cowley, J.-C. Gérard, A. Grocott, D. Grodent, L. Lamy, and P. Zarka (2010), Variation of 
Saturn's UV aurora with SKR phase, Geophys. Res. Lett., 37, L15102, doi:10.1029/2010GL044057.

Stallard, T., et al. (2007), Saturn's auroral/polar $H_{3}^{+}$infrared emission: I. General morphology and ion velocity structure, Icarus, 189(1), 1-13.

Stallard, T., et al. (2008), Complex structure within Saturn's infrared aurora, Nature, 456(7219), 214-217.

N. Achilleos, Atmospheric Physics Laboratory, Centre for Planetary Sciences, University College London, Gower Street, London WC1E 6BT, UK.
S. V. Badman, Institute of Space and Astronautical Science, Japan Aerospace Exploration Agency, 3-1-1 Yoshinodai, Chuo-ku, Sagamihara, Kanagawa 252-5210, Japan. (s.badman@stp.isas.jaxa.jp)

K. H. Baines, SSEC, University of Wisconsin-Madison, 1225 West Dayton St., Madison, WI 53706, USA.

R. H. Brown, Lunar and Planetary Laboratory, University of Arizona, Tucson, AZ 85721, USA.

E. J. Bunce, H. Melin, J. D. Nichols, and T. Stallard, Department of Physics and Astronomy, University of Leicester, University Road, Leicester LE1 7RH, UK.

M. K. Dougherty, Blackett Laboratory, Imperial College London, South Kensington Campus, London SW7 2AZ, UK. 